\title{
Product Gaussian quadrature on circular lunes*
}

\author{
Gaspare Da Fies and Marco Vianello ${ }^{1}$
}

August 30, 2013

\begin{abstract}
Resorting to recent results on subperiodic trigonometric quadrature, we provide three product Gaussian quadrature formulas exact on algebraic polynomials of degree $n$ on circular lunes. The first works on any lune, and has $n^{2}+\mathcal{O}(n)$ cardinality. The other two have restrictions on the lune angular intervals, but their cardinality is $n^{2} / 2+\mathcal{O}(n)$.
\end{abstract}

2000 AMS subject classification: 65D32.

Keywords: product Gaussian quadrature, subperiodic trigonometric quadrature, circular lunes.

\section{Introduction}

Quadrature problems concerning a circular lune, i.e., the portion of a disk not "obscured" by an overlapping disk, have a very long and fascinating history, starting from the famous quadrature of Hippocrate's lunes (5th century BC), passing through Clausen's and Euler's contributions in the 18th century, till the classification of the lunes that are constructible by compass and straightedge and that have equal area to a given square, eventually obtained in the mid-20th century by the Russian mathematicians Chebotaryov and Dorodnov via Galois theory; cf., e.g., [11, 15].

In the present paper, we study the problem of quadrature on lunes, that is numerical integration of a bivariate function on a circular lune, constructing three product Gaussian quadrature formulas that are exact on algebraic polynomials up to a given degree. Quite surprisingly, such a problem has not been yet addressed in the numerical literature, at least in the present

\footnotetext{
*Supported by the "ex-60\%" funds and by the biennial project CPDA124755 of the University of Padova, and by the GNCS-INdAM.

${ }^{1}$ Dept. of Mathematics, University of Padova, Italy e-mail: marcov@math.unipd.it
} 
formulation concerning polynomial exactness on the original domain. Some approaches have indeed been studied, for example in $[16,17]$, where however a preliminary polynomial or spline approximation of the lune boundary is needed.

The key of our approach is given by suitable trigonometric transformations, that map (the interior of) a rectangle (in angular variables) diffeomorphically onto (the interior of) the lune, and allow to resort to some recently developed trigonometric Gaussian rules on subintervals of the period (cf. [5]). In such a way we complete our study of algebraic cubature on the geometrical figures related to a couple of overlapping disks: lenses (intersection of two disks), double bubbles (union of two disks), cf. [4], and lunes (difference of two disks).

All these results, indeed, and many other concerning sections of the disk (circular segments, sectors, zones) and more generally integration domains related to circular and elliptical arcs, are based on the recently developed topic of "subperiodic" trigometric interpolation and quadrature; cf. $[2,3,5$, $6,10]$.

For the reader's convenience, we report the main result of [5], stated here for a general angular interval:

Proposition 1 Let $[\alpha, \beta]$ be an angular interval, with $0<\beta-\alpha \leq 2 \pi$. Let $\left\{\left(\xi_{j}, \lambda_{j}\right)\right\}_{1 \leq j \leq n+1}$, be the nodes and positive weights of the algebraic Gaussian quadrature formula for the weight function

$$
w(x)=\frac{2 \sin (\omega / 2)}{\sqrt{1-\sin ^{2}(\omega / 2) x^{2}}}, x \in(-1,1), \omega=\frac{\beta-\alpha}{2} \leq \pi .
$$

Then

$$
\int_{\alpha}^{\beta} t(\theta) d \theta=\sum_{j=1}^{n+1} \lambda_{j} t\left(\theta_{j}\right),
$$

for every trigonometric polynomial $t \in \mathbb{T}_{n}([\alpha, \beta])$, where

$$
\theta_{j}=\frac{\alpha+\beta}{2}+2 \arcsin \left(\xi_{j} \sin \left(\frac{\omega}{2}\right)\right) \in(\alpha, \beta), j=1,2, \ldots, n+1 .
$$

Observe that, since the weight function (1) is even, the set of angular nodes is symmetric with respect to the center of the interval, and that symmetric nodes have equal weight, cf. [8].

The paper is organized as follows. In Sections 2 and 3 we develop the theoretical construction of three product Gaussian quadrature formulas exact on algebraic polynomials of degree $n$ on circular lunes. The first works on any lune, and has $n^{2}+\mathcal{O}(n)$ cardinality. The other two have restrictions on the lune angular intervals, but their cardinality is $n^{2} / 2+\mathcal{O}(n)$. Moreover, we discuss the convergence rate of such formulas in connection with multivariate Jackson inequality. 
In Section 4, we present some numerical results obtained by a Matlab implementation of the product quadrature formulas. The corresponding codes are available online in [7]. We do not develop specific applications here, but it is worth recalling that Gaussian quadrature on domains defined by circular arcs is of practical interest, for example, in the field of optical design and optimization; cf. [1].

\section{A general transformation}

In this section we construct a basic product quadrature formula, which is valid on any circular lune. By no loss of generality, up to rotation, translation and scaling, we can consider a lune, say $\mathcal{L}$, which is the difference of the unit disk with of a disk of radius $r$ centered at $(-d, 0), d>0$. The condition $|1-r|<d<1+r$ ensures that the intersection is nonempty and one disk is not included into the other, so that we have a proper lune. The boundary of the lune is given by two circular arcs: the right (longer) is an arc of the unit disk with semiangle $\omega_{2}$, the left (shorter) is an arc of the other disk with semiangle $\omega_{1}$, where

$$
0<\omega_{1}=\arccos \left(\frac{r^{2}+d^{2}-1}{2 d r}\right)<\omega_{2}=\arccos \left(\frac{r^{2}-d^{2}-1}{2 d}\right)<\pi .
$$

Examples of lunes as difference of overlapping disks can be seen in Figures 3 and 4 below.

We now try to construct a transformation $P: \mathcal{R} \rightarrow \mathcal{L}$ of the rectangle

$$
\mathcal{R}=\left[-\omega_{1}, \omega_{1}\right] \times\left[\omega_{1}, \omega_{2}\right]
$$

onto the lune $\mathcal{L}, P(\phi, \theta)=(x(\phi, \theta), y(\phi, \theta))$, of the form

$$
\begin{gathered}
P(\phi, \theta)=A_{1}+A_{2} \cos (\theta)+A_{3} \sin (\theta)+A_{4} \cos (\phi)+A_{5} \cos (\phi) \cos (\theta) \\
+A_{6} \cos (\phi) \sin (\theta)+A_{7} \sin (\phi)+A_{8} \sin (\phi) \cos (\theta)+A_{9} \sin (\phi) \sin (\theta)
\end{gathered}
$$

where the $A_{i}=\left(A_{i}(1), A_{i}(2)\right)$ are suitable 2 -dimensional vectors, i.e., each component of $P$ is in the trigonometric space $\mathbb{T}_{1} \otimes \mathbb{T}_{1}$.

The idea is that for $\theta=\omega_{1}$ the corresponding curve is the lune's right boundary subarc $(\cos (\phi), \sin (\phi))$, for $\theta=\omega_{2}$ it is the left boundary sub$\operatorname{arc}(-d, 0)+r(\cos (\phi), \sin (\phi))$, for $\phi=\omega_{1}$ it is the right boundary subarc $(\cos (\theta), \sin (\theta))$. Moreover, we impose that, for fixed $\theta$, varying $\phi$ we have circular arcs, symmetric with respect to the $x$-axis. After simple but lengthy calculations (not reported for brevity) we obtain

$$
x(\phi, \theta)=\cos (\theta)-\frac{\cos \left(\omega_{1}\right)}{\sin \left(\omega_{1}\right)} \sin (\theta)+\frac{1}{\sin \left(\omega_{1}\right)} \cos (\phi) \sin (\theta),
$$




$$
y(\phi, \theta)=\frac{1}{\sin \left(\omega_{1}\right)} \sin (\phi) \sin (\theta)
$$

with Jacobian

$$
\operatorname{det}(J P(\phi, \theta))=\frac{\sin (\theta)\left(\cos (\phi) \cos \left(\theta-\omega_{1}\right)-\cos (\theta)\right.}{\sin ^{2}\left(\omega_{1}\right)} .
$$

Since the boundary of the rectangle is mapped onto the boundary of the lune (preserving the orientation) and the Jacobian is positive in the interior, this transformation is a diffeomorphism that maps the interior of the rectangle onto the interior of the lune. Indeed, $\sin (\theta) / \sin ^{2}\left(\omega_{1}\right)>0$ because $0<\omega_{1} \leq$ $\theta \leq \omega_{2}<\pi$, thus we only have to check the sign of the second factor in (6). Now, $\cos (\phi) \geq \cos \left(\omega_{1}\right)$, which gives

$$
\begin{gathered}
\cos (\phi) \cos \left(\theta-\omega_{1}\right)-\cos (\theta) \geq \cos \left(\omega_{1}\right) \cos \left(\theta-\omega_{1}\right)-\cos (\theta) \\
=\cos \left(\theta-\omega_{1}+\omega_{1}\right)+\sin \left(\theta-\omega_{1}\right) \sin \left(\omega_{1}\right)-\cos (\theta)=\sin \left(\theta-\omega_{1}\right) \sin \left(\omega_{1}\right)>0
\end{gathered}
$$

for $\theta>\omega_{1}$.

The geometric action of transformation (5) can be seen in Figure 1-left.

We are now ready to state and prove the following

Proposition 2 Let $\mathcal{L}$ be the circular lune obtained as difference of the unit disk with a disk of radius $r$ centered at $(-d, 0), d>0$ (cf. (3)), and let $P$ be the transformation (4)-(6). The following product Gaussian formula holds

$$
\iint_{\mathcal{L}} f(x, y) d x d y=\sum_{i=1}^{n+2} \sum_{j=1}^{n+3} W_{i j} f\left(x_{i j}, y_{i j}\right), \quad \forall f \in \mathbb{P}_{n}^{2}
$$

where $\mathbb{P}_{n}^{2}$ denotes the space of bivariate polynomials of total degree not greater than $n$, and

$$
W_{i j}=\operatorname{det}\left(J P\left(\phi_{i}, \theta_{j}\right)\right) \lambda_{i} \mu_{j}, \quad\left(x_{i j}, y_{i j}\right)=P\left(\phi_{i}, \theta_{j}\right),
$$

$\left\{\phi_{i}\right\},\left\{\lambda_{i}\right\}$ being the angles and weights of the trigonometric Gaussian formula (2) of degree of exactness $n+1$ on $\left[-\omega_{1}, \omega_{1}\right]$, and $\left\{\theta_{j}\right\},\left\{\mu_{j}\right\}$ those of the trigonometric Gaussian formula (2) of degree of exactness $n+2$ on $\left[\omega_{1}, \omega_{2}\right]$.

Proof. Since the transformation $P: \stackrel{\circ}{\mathcal{R}} \rightarrow \stackrel{\circ}{\mathcal{L}}$ is a diffeomorphism with positive Jacobian, we can apply the change of variables theorem for double integration, obtaining

$$
\iint_{\mathcal{L}} f(x, y) d x d y=\int_{-\omega_{1}}^{\omega_{1}} \int_{\omega_{1}}^{\omega_{2}} f(P(\phi, \theta)) \operatorname{det}(J P(\phi, \theta)) d \theta d \phi .
$$


Now, $f(x, y) \in \mathbb{P}_{n}^{2}$ implies that $f(P(\phi, \theta)) \operatorname{det}(J P(\phi, \theta))$ belongs to the trigonometric tensor-product space $\mathbb{T}_{n+1}\left(\left[-\omega_{1}, \omega_{1}\right]\right) \otimes \mathbb{T}_{n+2}\left(\left[\omega_{1}, \omega_{2}\right]\right)$, so that we get exactness using the corresponding trigonometric Gaussian formula (2) in each variable.

Observe that the cardinality of the product Gaussian formula (7) is approximately $(n+2)(n+3)=n^{2}+\mathcal{O}(n)$.

\section{Reducing the number of nodes}

In order to reduce the number of quadrature nodes, we can try to use axial symmetry, together with symmetry of subperiodic trigonometric Gaussian quadrature, in the spirit of the construction of [4] for circular segments. We have studied two transformations, which lead to formulas with substantially half the cardinality of (7), but have restrictions on the lune's angular interval.

\subsection{First approach}

We seek a transformation $P: \mathcal{R}^{\prime} \rightarrow \mathcal{L}$ of the form (4), where

$$
\mathcal{R}^{\prime}=\left[-\omega_{1}, \omega_{1}\right] \times\left[0, \omega_{2}\right],
$$

such that, $P(\phi, 0) \equiv 1$, for $\theta=\omega_{2}$ it gives the lune's left boundary subarc $(-d, 0)+r(\cos (\phi), \sin (\phi))$, for $\phi=\omega_{1}$ it gives the right boundary subarc $(\cos (\theta), \sin (\theta))$. By imposing symmetry with respect to the $x$-axis, $x(\phi, \theta)=x(-\phi, \theta)$ and $y(\phi, \theta)=-y(-\phi, \theta)$, together with the further symmetry condition $x(\phi, \theta)=x(\phi,-\theta)$ and $y(\phi, \theta)=-y(\phi,-\theta)$, we eventually obtain

$$
\begin{gathered}
x(\phi, \theta)=\cos (\theta)+\frac{(1-\cos (\theta)) \sin \left(\omega_{2}\right)}{\left(1-\cos \left(\omega_{2}\right)\right) \sin \left(\omega_{1}\right)}\left(\cos (\phi)-\cos \left(\omega_{1}\right)\right), \\
y(\phi, \theta)=\frac{1}{\sin \left(\omega_{1}\right)} \sin (\phi) \sin (\theta),
\end{gathered}
$$

with Jacobian

$$
\operatorname{det}(J P(\phi, \theta))=\frac{g(\phi, \theta)}{\sin ^{2}\left(\omega_{1}\right)\left(1-\cos \left(\omega_{2}\right)\right)},
$$

where

$$
\begin{gathered}
g(\phi, \theta)=\left(\left(1-\cos \left(\omega_{2}\right)\right) \sin \left(\omega_{1}\right)+\cos \left(\omega_{1}\right) \sin \left(\omega_{2}\right)\right) \sin ^{2}(\theta) \cos (\phi) \\
-\sin \left(\omega_{2}\right) \sin ^{2}(\theta) \cos ^{2}(\phi)-\sin \left(\omega_{2}\right) \sin ^{2}(\phi) \cos (\theta)+\sin \left(\omega_{2}\right) \sin ^{2}(\phi) \cos ^{2}(\theta) .
\end{gathered}
$$


This transformation maps the boundary of the rectangle $\mathcal{R}^{\prime}$ onto the boundary of the lune, non injectively since one side collapses into one single point, $P(\phi, 0) \equiv(1,0)$, and preserving the orientation, so in order to check whether it is a diffeomorphism that maps the interior of the rectangle onto the interior of the lune, we have to study under which conditions the Jacobian is positive (i.e., $g(\theta, \phi)$ is positive) in the interior.

It is not difficult to show that $\operatorname{det}(J P(\phi, \theta))$ is positive in $\stackrel{\circ}{\mathcal{R}}^{\prime}$ if and only if the following inequality holds

$$
\omega_{1} \leq \arctan \left(\frac{2\left(1-\cos \left(\omega_{2}\right)\right)}{\sin \left(\omega_{2}\right)}\right) .
$$

To prove that condition (13) is necessary, consider the function $g(\phi, \theta)$ in (12) restricted to a portion of the boundary, $\phi=\omega_{1}$. Now,

$$
\begin{gathered}
g\left(\omega_{1}, \theta\right)=\left(1-\cos \left(\omega_{2}\right)\right) \sin \left(\omega_{1}\right) \cos \left(\omega_{1}\right) \sin ^{2}(\theta) \\
-\sin \left(\omega_{2}\right) \sin ^{2}\left(\omega_{1}\right) \cos (\theta)(1-\cos (\theta))
\end{gathered}
$$

is clearly negative for $\omega_{1} \geq \pi / 2$ and $0<\theta<\pi / 2$. On the other hand, if (13) is not satisfied

$$
\lim _{\theta \rightarrow 0^{+}} \frac{g\left(\omega_{1}, \theta\right)}{\sin ^{2}(\theta)}=\left(1-\cos \left(\omega_{2}\right)\right) \sin \left(\omega_{1}\right) \cos \left(\omega_{1}\right)-\frac{1}{2} \sin \left(\omega_{2}\right) \sin ^{2}\left(\omega_{1}\right)<0,
$$

and thus $g\left(\omega_{1}, \theta\right)<0$ in a neighborhood of $\theta=0$.

In order to prove that (13) is sufficient, we write the estimate

$$
\begin{gathered}
\frac{1}{\sin \left(\omega_{2}\right)} g(\phi, \theta)=\left(\left(1-\cos \left(\omega_{2}\right)\right) \frac{\sin \left(\omega_{1}\right)}{\sin \left(\omega_{2}\right)}+\cos \left(\omega_{1}\right)-\cos (\phi)\right) \sin ^{2}(\theta) \cos (\phi) \\
-\sin ^{2}(\phi) \cos (\theta)(1-\cos (\theta)) \geq\left(\tan \left(\omega_{1}\right) \sin \left(\omega_{1}\right) / 2+\cos \left(\omega_{1}\right)\right. \\
-\cos (\phi)) \sin ^{2}(\theta) \cos (\phi)-\sin ^{2}(\phi) \cos (\theta)(1-\cos (\theta)),
\end{gathered}
$$

and thus $\operatorname{det}(J P(\phi, \theta))$ is positive if the last trigonometric expression above is positive, i.e.,

$$
\frac{1+\cos ^{2}\left(\omega_{1}\right)-2 \cos \left(\omega_{1}\right) \cos (\phi)}{2 \cos \left(\omega_{1}\right)} \frac{\cos (\phi)}{\sin ^{2}(\phi)}>\frac{\cos (\theta)(1-\cos (\theta))}{\sin ^{2}(\theta)} .
$$

For $\theta>\pi / 2,(14)$ holds clearly true, because the left-hand side is positive and the right-hand side is negative, under our assumption that $-\pi / 2<-\omega_{1} \leq \phi \leq \omega_{1}<\pi / 2$. For $0<\theta \leq \pi / 2$ we have $0 \leq \cos (\theta)(1-$ $\cos (\theta)) / \sin ^{2}(\theta)<1 / 2$, and thus it is sufficient to show that

$$
\frac{1+\cos ^{2}\left(\omega_{1}\right)-2 \cos \left(\omega_{1}\right) \cos (\phi)}{2 \cos \left(\omega_{1}\right)} \frac{\cos (\phi)}{\sin ^{2}(\phi)} \geq \frac{1}{2} .
$$


Also the latter inequality holds true, since the left-hand side has global minimum at $\phi= \pm \omega_{1}$ and this minimum is $1 / 2$.

The geometric action of transformation (10) can be seen in Figure 1center, where the lune satisfies (13).

Figure 1: Mapping a $10 \times 10$ angular grid from the relevant rectangles to a lune by the transformations (5) (left), (10) (center) and (18) (right).
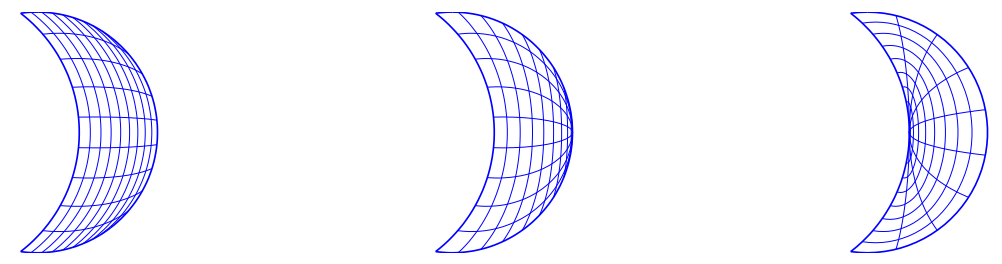

At this point, we can state the following result on the construction of product Gaussian quadrature with halved cardinality.

Proposition 3 Let $\mathcal{L}$ be the circular lune obtained as difference of the unit disk with a disk of radius $r$ centered at $(-d, 0), d>0$ (cf. (3)), where the angular intervals satisfy (13), and let $P$ be the transformation (10)-(12). The following product Gaussian formula holds

$$
\iint_{\mathcal{L}} f(x, y) d x d y=\sum_{i=1}^{n+3} \sum_{j=1}^{\left\lceil\frac{n+2}{2}\right\rceil} W_{i j} f\left(x_{i j}, y_{i j}\right), \quad \forall f \in \mathbb{P}_{n}^{2},
$$

where $\mathbb{P}_{n}^{2}$ denotes the space of bivariate polynomials of total degree not greater than $n$, and

$$
W_{i j}=\operatorname{det}\left(J P\left(\phi_{i}, \theta_{j}\right)\right) \lambda_{i} \mu_{j}, \quad\left(x_{i j}, y_{i j}\right)=P\left(\phi_{i}, \theta_{j}\right),
$$

$\left\{\phi_{i}\right\},\left\{\lambda_{i}\right\}$ being the angles and weights of the trigonometric Gaussian formula (2) of degree of exactness $n+2$ on $\left[-\omega_{1}, \omega_{1}\right]$, and $\left\{\theta_{j}\right\},\left\{\mu_{j}\right\}$ those of the trigonometric Gaussian formula (2) of degree of exactness $n+2$ on $\left[-\omega_{2}, \omega_{2}\right]$.

Proof. Since under assumption (13) the transformation $P: \stackrel{\circ}{\mathcal{R}^{\prime}} \rightarrow \stackrel{\circ}{\mathcal{L}}$ is a diffeomorphism with positive Jacobian, we can apply the change of variables theorem for double integration, obtaining

$$
\iint_{\mathcal{L}} f(x, y) d x d y=\int_{-\omega_{1}}^{\omega_{1}} \int_{0}^{\omega_{2}} f(P(\phi, \theta)) \operatorname{det}(J P(\phi, \theta)) d \theta d \phi .
$$


On the other hand, by symmetry of the transformation we can also write

$$
\iint_{\mathcal{L}} f(x, y) d x d y=\int_{-\omega_{1}}^{\omega_{1}} \int_{-\omega_{2}}^{0} f(P(\phi, \theta)) \operatorname{det}(J P(\phi, \theta)) d \theta d \phi,
$$

which together with the former gives

$$
\iint_{\mathcal{L}} f(x, y) d x d y=\frac{1}{2} \int_{-\omega_{1}}^{\omega_{1}} \int_{-\omega_{2}}^{\omega_{2}} f(P(\phi, \theta)) \operatorname{det}(J P(\phi, \theta)) d \theta d \phi .
$$

Now, $f(x, y) \in \mathbb{P}_{n}^{2}$ implies that $f(P(\phi, \theta)) \operatorname{det}(J P(\phi, \theta))$ belongs to the trigonometric tensor-product space $\mathbb{T}_{n+2}\left(\left[-\omega_{1}, \omega_{1}\right]\right) \otimes \mathbb{T}_{n+2}\left(\left[-\omega_{2}, \omega_{2}\right]\right)$. Thus, using the corresponding trigonometric Gaussian formula (2) in each variable, and observing that by symmetry each node is repeated twice with equal weight, apart from the node $(1,0)$ for $n$ even, which is repeated $2(n+3)$ times but has null weight, we obtain the product Gaussian formula (15)-(16).

Observe that the cardinality of the product Gaussian formula (15) is approximately $(n+2)(n+3) / 2=n^{2} / 2+\mathcal{O}(n)$, that is half the cardinality of (7). In particular, it is exactly half for $n$ even, and $(n+3)^{2} / 2$ for $n$ odd.

\subsection{Second approach}

We seek a transformation $P: \mathcal{R}^{\prime \prime} \rightarrow \mathcal{L}$ of the form (4), where

$$
\mathcal{R}^{\prime \prime}=\left[0, \omega_{1}\right] \times\left[-\omega_{2}, \omega_{2}\right],
$$

such that, $P(0, \theta) \equiv(-d+r, 0)$, for $\theta=\omega_{2}$ it gives the lune's left boundary subarc $(-d, 0)+r(\cos (\phi), \sin (\phi)), 0 \leq \phi \leq \omega_{1}$, for $\phi=\omega_{1}$ it gives the right boundary subarc $(\cos (\theta), \sin (\theta))$. By imposing symmetry with respect to the $x$-axis, $x(\phi, \theta)=x(\phi,-\theta)$ and $y(\phi, \theta)=-y(\phi,-\theta)$, together with the further symmetry condition $x(\phi, \theta)=x(-\phi, \theta)$ and $y(\phi, \theta)=-y(-\phi, \theta)$, we eventually obtain

$$
\begin{gathered}
x(\phi, \theta)=\left(\cos (\phi)-\cos \left(\omega_{1}\right)\right)\left(\frac{\cos \left(\omega_{2}\right)}{1-\cos \left(\omega_{1}\right)}+\frac{\sin \left(\omega_{2}\right)}{\sin \left(\omega_{1}\right)}\right)+\frac{1-\cos (\phi)}{1-\cos \left(\omega_{1}\right)} \cos (\theta), \\
y(\phi, \theta)=\frac{1}{\sin \left(\omega_{1}\right)} \sin (\phi) \sin (\theta)
\end{gathered}
$$

with Jacobian

$$
\operatorname{det}(J P(\phi, \theta))=\frac{g(\phi, \theta)}{\sin \left(\omega_{1}\right)\left(1-\cos \left(\omega_{1}\right)\right)},
$$

where

$$
g(\phi, \theta)=-\left(\cos \left(\omega_{2}\right)+\frac{\sin \left(\omega_{2}\right)}{\sin \left(\omega_{1}\right)}\left(1-\cos \left(\omega_{1}\right)\right)\right) \sin ^{2}(\phi) \cos (\theta)+\sin ^{2}(\phi) \cos ^{2}(\theta)
$$




$$
+\cos (\phi) \sin ^{2}(\theta)-\cos ^{2}(\phi) \sin ^{2}(\theta) .
$$

Also this transformation, as that in (10), maps the boundary of the rectangle $\mathcal{R}^{\prime \prime}$ onto the boundary of the lune, non injectively since one side collapses into one single point, $P(0, \theta) \equiv(-d+r, 0)$, and preserves the boundary orientation. In order to check whether it is a diffeomorphism that maps the interior onto the interior, with the same reasoning made for the previous transformation we have to check under which conditions the Jacobian is positive (i.e., $g(\phi, \theta)$ is positive) in the interior.

We prove now that $\operatorname{det}(J P(\phi, \theta))$ is positive in $\mathcal{R}^{\prime \prime}$ if and only if the following inequality holds

$$
\left(\cos \left(\omega_{2}\right)+\cos \left(\omega_{2}-\omega_{1}\right)\right)^{2} \leq 4 \cos \left(\omega_{1}\right) .
$$

Observe that condition (21) determines a set of the parameters $\omega_{1}, \omega_{2}$ which has nonempty difference with that determined by condition (13); see Figure 2, where (13) corresponds to the area below the dashed curve, and (21) to that below the solid curve. Notice that, as (13), also (21) implies that $\omega_{1}<\pi / 2$, except for the tangency point $\left(\omega_{2}, \omega_{1}\right)=\left(\frac{3 \pi}{4}, \frac{\pi}{2}\right)$. In the region above both the curves, and in particular for $\omega_{1}>\pi / 2$, only the general formula (7) is applicable.

To prove that condition (21) is necessary, consider

$$
\frac{g\left(\omega_{1}, \theta\right)}{1-\cos \left(\omega_{1}\right)}=-\left(\cos \left(\omega_{2}\right)+\cos \left(\omega_{2}-\omega_{1}\right)\right) \cos (\theta)+\cos ^{2}(\theta)+\cos \left(\omega_{1}\right),
$$

which has the same sign of $\operatorname{det}\left(J P\left(\omega_{1}, \theta\right)\right)$. It is not difficult to check that such a function has global minimum for $\theta= \pm \theta^{*}$ where $0<\theta^{*}=$ $\arccos \left(\left(\cos \left(\omega_{2}\right)+\cos \left(\omega_{2}-\omega_{1}\right)\right) / 2\right)<\omega_{2}$, and the condition

$$
\frac{g\left(\omega_{1}, \theta^{*}\right)}{1-\cos \left(\omega_{1}\right)}=-\left(\cos \left(\omega_{2}\right)+\cos \left(\omega_{2}-\omega_{1}\right)\right)^{2} / 4+\cos \left(\omega_{1}\right) \geq 0
$$

is exactly $(21)$.

To prove that condition (21) is sufficient for positivity of the Jacobian in the interior, since the denominator in (19) is positive, we are reduced to prove that $g(\phi, \theta)$ is positive. Now,

$$
\frac{\partial g}{\partial \phi}=\sin (\phi)\left(2 a \cos (\phi)-\sin ^{2}(\theta)\right),
$$

where

$$
a=1-\left(\cos \left(\omega_{2}\right)+\cos \left(\omega_{2}-\omega_{1}\right)\right) \frac{1-\cos \left(\omega_{1}\right)}{\sin ^{2}\left(\omega_{1}\right)} .
$$

Since by (21)

$$
\left|\cos \left(\omega_{2}\right)+\cos \left(\omega_{2}-\omega_{1}\right)\right| \frac{1-\cos \left(\omega_{1}\right)}{\sin ^{2}\left(\omega_{1}\right)} \leq 2 \sqrt{\cos \left(\omega_{1}\right)} \frac{1-\cos \left(\omega_{1}\right)}{\sin ^{2}\left(\omega_{1}\right)}<1,
$$


we have $a>0$ and $2 a \cos (\phi)-\sin ^{2}(\theta)$ is a strictly decreasing function of $\phi \in\left[0, \omega_{1}\right]$. Now, $\operatorname{sign}(\partial g / \partial \phi)=\operatorname{sign}\left(2 a \cos (\phi)-\sin ^{2}(\theta)\right)$ because $\sin (\phi) \geq 0$ in $\left(0, \omega_{1}\right]$, thus $\partial g / \partial \phi$ may change sign at most once, i.e., either it doesn't change sign, or it may change from positive to negative increasing $\phi$ (it cannot change from negative to positive, the factor $2 a \cos (\phi)-\sin ^{2}(\theta)$ being strictly decreasing in $\phi)$. This entails that, for fixed $\theta$, the function $g(\phi, \theta)$ takes its minimum either at $\phi=0$ (where it vanishes) or at $\phi=\omega_{1}$, and we have proved above that nonnegativity of $g\left(\omega_{1}, \theta\right)$ is equivalent to $(21)$.

The geometric action of transformation (18) can be seen in Figure 1right, where the lune satisfies (21).

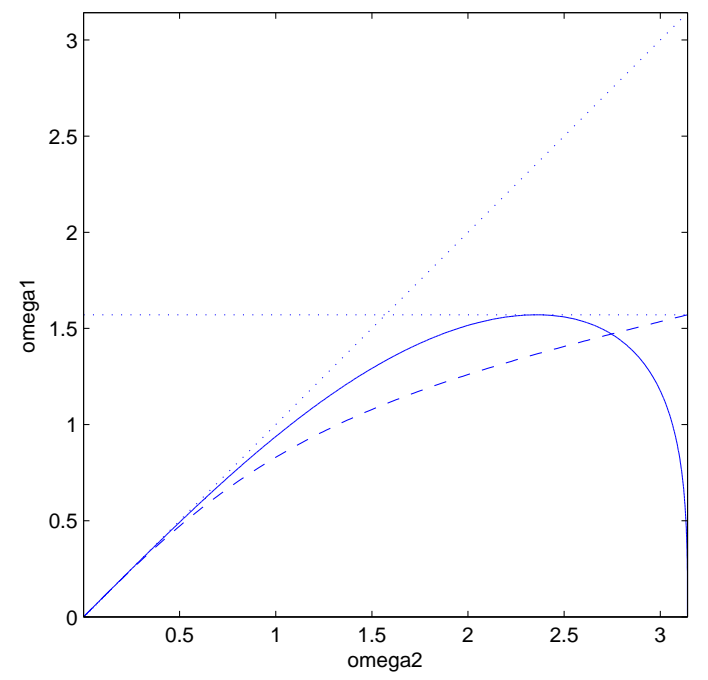

Figure 2: Regions of applicability of the quadrature formulas for a lune in the $\left(\omega_{2}, \omega_{1}\right)$ plane (see (13) and (21)): formula (15)-(16) can be applied below the dashed curve, formula (22)-(23) below the solid curve, formula (7)-(8) for every pair of angles (3); the horizontal dotted line is $\omega_{1}=\pi / 2$.

We can give now a statement concerning the third product Gaussian formula.

Proposition 4 Let $\mathcal{L}$ be the circular lune obtained as difference of the unit disk with a disk of radius $r$ centered at $(-d, 0), d>0$ (cf. (3)), where the angular intervals satisfy (21), and let $P$ be the transformation (18)-(20). The following product Gaussian formula holds

$$
\iint_{\mathcal{L}} f(x, y) d x d y=\sum_{i=1}^{\left\lceil\frac{n+2}{2}\right\rceil} \sum_{j=1}^{n+3} W_{i j} f\left(x_{i j}, y_{i j}\right), \quad \forall f \in \mathbb{P}_{n}^{2},
$$


where $\mathbb{P}_{n}^{2}$ denotes the space of bivariate polynomials of total degree not greater than $n$, and

$$
W_{i j}=\operatorname{det}\left(J P\left(\phi_{i}, \theta_{j}\right)\right) \lambda_{i} \mu_{j}, \quad\left(x_{i j}, y_{i j}\right)=P\left(\phi_{i}, \theta_{j}\right),
$$

$\left\{\phi_{i}\right\},\left\{\lambda_{i}\right\}$ being the angles and weights of the trigonometric Gaussian formula (2) of degree of exactness $n+2$ on $\left[-\omega_{1}, \omega_{1}\right]$, and $\left\{\theta_{j}\right\},\left\{\mu_{j}\right\}$ those of the trigonometric Gaussian formula (2) of degree of exactness $n+2$ on $\left[-\omega_{2}, \omega_{2}\right]$.

The proof of proposition 4 proceeds as that of Proposition 3, with the roles of $\omega_{1}$ and $\omega_{2}$ interchanged. This time it is the node $(-d+r, 0)$ to be repeated $2(n+3)$ times, but it has null weight so it doesn't appear in (22). Observe that, as (15), also the product Gaussian formula (22) has approximately $(n+2)(n+3) / 2=n^{2} / 2+\mathcal{O}(n)$ nodes, that is half the cardinality of (7).

Remark 1 (Convergence rate). Let $I_{n}(f)$ be any of the three product Gaussian formulas discussed above. Concerning the convergence rate, due to the positivity of the weights it is simple to show by standard arguments of quadrature theory that

$$
\begin{aligned}
\mid \iint_{\mathcal{L}} f(x, y) d x d y & -I_{n}(f) \mid \leq\left(\operatorname{meas}(\mathcal{L})+\sum W_{i j}\right) \operatorname{dist}_{\mathcal{L}}\left(f, \mathbb{P}_{n}^{2}\right) \\
& =2 \operatorname{meas}(\mathcal{L}) \operatorname{dist}_{\mathcal{L}}\left(f, \mathbb{P}_{n}^{2}\right)
\end{aligned}
$$

which implies that for any fixed $k>0$ and sufficiently regular integrand $f$, we have the error estimate

$$
\iint_{\mathcal{L}} f(x, y) d x d y=I_{n}(f)+\mathcal{O}\left(n^{-k}\right)
$$

since a lune is a Jackson compact, cf. [14].

We recall that a fat compact set $\Omega \subset \mathbb{R}^{d}$ (i.e., $\Omega=\overline{\Omega^{\circ}}$ ) is termed a Jackson compact if it admits a Jackson inequality, namely for each $k \in \mathbb{N}$ there exist a positive integer $m_{k}$ and a positive constant $c_{k}$ such that

$$
n^{k} \operatorname{dist}_{\Omega}\left(f, \mathbb{P}_{n}^{d}\right) \leq c_{k} \sum_{|i| \leq m_{k}}\left\|D^{i} f\right\|_{\Omega}, n>k, \quad \forall f \in C^{m_{k}}(\Omega)
$$

where

$$
\operatorname{dist}_{\Omega}\left(f, \mathbb{P}_{n}^{d}\right)=\inf \left\{\|f-p\|_{\infty, \Omega}, p \in \mathbb{P}_{n}^{d}\right\} .
$$

Examples of Jackson compacts are $d$-dimensional cubes (with $m_{k}=k+1$ ) and Euclidean balls (with $m_{k}=k$ ). 
In [14], it is proved that any fat Whitney regular Markov compact is a Jackson compact. Now, a lune has both these properties, being the image of a rectangle by the analytic transformation (5) and thus a sub-analytic set (cf. $[13,18]$ concerning sub-analytic geometry).

More directly, a lune admits a Markov polynomial inequality with exponent 2 because it is a Lipschitz domain, i.e., at the boundary it is locally the cartesian graph of a Lipschitz continuous function. This is due to the fact that the circles are not tangent at the lune's corners. Moreover, a lune is Whitney 1-regular, i.e., any pair of points can be joined by an internal curve whose length is bounded by their Euclidean distance times a fixed factor. Indeed, depending on their position inside the lune, two points can be joined either by the corresponding segment if it doesn't intersect the lune's shorter side, or by the curve formed by two pieces of the segment joined by the subarc of such a side between the intersection points. In any case, it is not difficult to realize that the ratio (curve length)/distance is bounded by $r \omega_{1} / \sin \left(\omega_{2}\right)$.

\section{Implementation and examples}

Efficient implementation of the product Gaussian formulas (7), (15) and (22) on circular lunes, is clearly based on fast computation of the trigonometric Gaussian formula (2). A Matlab implementation of the latter is provided in [3], by exploiting fast and stable computation of modified Chebyshev moments and the algorithms for orthogonal polynomials in the OPQ suite [9] by W. Gautschi. It is worth recalling that Gaussian quadrature for the weight function (1), can also be embedded in the more general framework of "sub-range" Jacobi polynomials, cf. [10].

In [7] we provide a Matlab code, named gqlune, that implements the three product quadrature formulas studied in the present paper, for a general lune. It can be applied to the difference of any disk with any other disk (with arbitrary centers and radii), recognizing automatically whether the lune is proper, and applying different quadrature formulas in the degenerate case (that is when the disks do not overlap) or the second disk is contained into the first (in this latter case the region is treated as an asymmetric annulus, via the approach of [3]).

For a proper lune, by default the code chooses automatically the lower cardinality formulas when (13) or (21) are satisfied (privileging (15) in the parameters intersection zone, see Figure 2), resorting to the general formula (7) otherwise.

Concerning efficiency of the method, using the computational tricks for trigonometric Gaussian quadrature discussed in [6], in particular a fast version of the Golub-Welsh algorithm, recently proposed in [12] for Gaussian rules with symmetric weight function, we are able to compute the product 
Gaussian formulas for lunes in just $10^{-2}$ seconds up to exactness degree $n=200$, that is up to tens of thousands nodes and weights.

All the numerical tests have been made in Matlab 7.7.0 with an Athlon 64 X2 Dual Core $4400+2.40 \mathrm{GHz}$ processor.

Example 1. We consider the lune obtained as difference of the disk centered in $(0,0)$ and radius 2 , with the disk centered in $(-1.8,0)$ and radius 2.5 ; the product quadrature nodes with exactness degree $n=4$ for the three formulas above are shown in Figure 3-left and Figure 4.

In Figure 5-top we show the relative errors obtained by the three product quadrature formulas on the slowly varying Gaussian

$$
f_{1}(x, y)=\exp \left[-\left((x-1)^{2}+(y-1)^{2}\right)\right],
$$

and the more rapidly varying Gaussian

$$
f_{2}(x, y)=\exp \left[-100\left((x-1)^{2}+(y-1)^{2}\right)\right] ;
$$

observe that the Gaussian center $(1,1)$ belongs to the interior of the lune. Such errors are plotted in loglog scale, with respect to the number of nodes, corresponding to the sequence of exactness degrees $n=5,10,15, \ldots, 95,100$. The reference value of the integrals has been obtained by the standard Matlab dblquad numerical integrator, with a relative error tolerance of $10^{-15}$ (notice that this requires a computational time in the order of the minutes, whereas we can obtain a comparable precision in few $10^{-2}$ seconds).

Example 2. We take the same lune as in Example 1, but this time we apply the product quadrature formulas to the $C^{0}$ function

$$
\left.f_{3}(x, y)=\left[(x-1)^{2}+(y-1)^{2}\right)\right]^{1 / 2},
$$

which has a singularity of the first derivatives at the internal point $(1,1)$, and to the $C^{4}$ function

$$
\left.f_{4}(x, y)=\left[(x-1)^{2}+(y-1)^{2}\right)\right]^{5 / 2},
$$

which has a singularity of the fifth derivatives at the same point. The loglog plot of the errors is shown in Figure 5-bottom.

Notice that, as expected in view of the cardinality for the same exactness degree, in both the Examples formulas (15) and (22) perform better than formula (7), in terms of precision as a function of the number of nodes (i.e., in terms of function evaluations). 
Figure 3: The $6 \times 7=42$ nodes of the product quadrature formula (7) with exactness degree $n=4$ on two circular lunes, with $\omega_{1}=0.9147, \omega_{2}=1.7987$ (left), and $\omega_{1}=1.5675, \omega_{2}=2.5526$ (right).
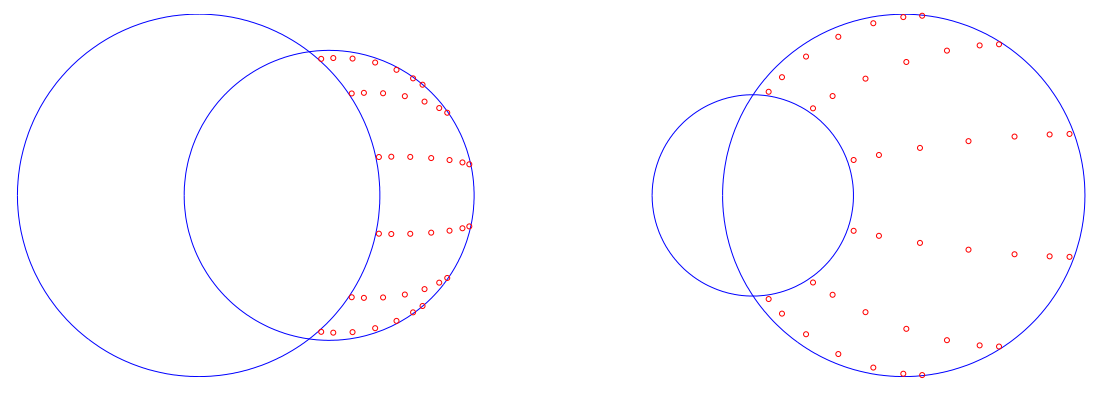

Figure 4: The $7 \times 3=21$ nodes of the product quadrature formulas (15) (left) and (22) (right), with exactness degree $n=4$ on the same circular lune of Figure 3-left.
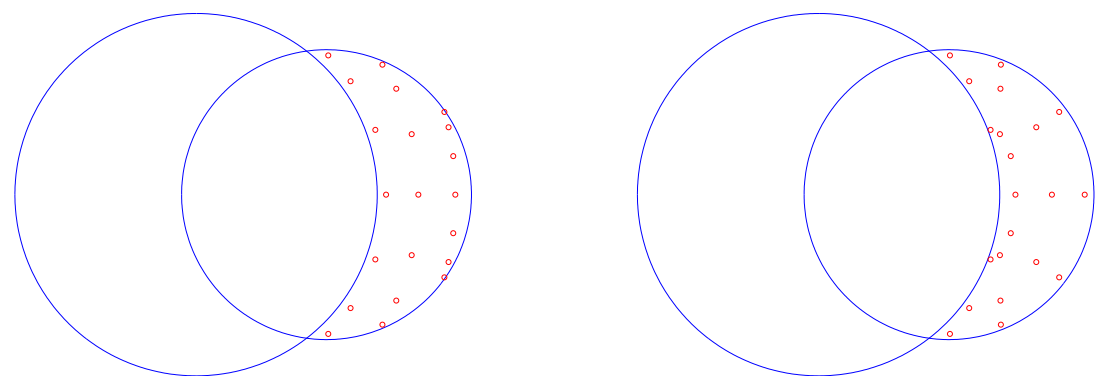

\section{References}

[1] B. Bauman and H. Xiao, Gaussian quadrature for optical design with noncircular pupils and fields, and broad wavelength range, Proc. SPIE, 7652(2) (2010), 1-12.

[2] L. Bos and M. Vianello, Subperiodic trigonometric interpolation and quadrature, Appl. Math. Comput. 218 (2012), 10630-10638.

[3] G. Da Fies, A. Sommariva and M. Vianello, Algebraic cubature by linear blending of elliptical arcs, Appl. Numer. Math, published online 21 August 2013.

[4] G. Da Fies and M. Vianello, Algebraic cubature on planar lenses and bubbles, Dolomites Res. Notes Approx. 5 (2012), 7-12. 
Figure 5: Relative quadrature errors (loglog scale) on the functions of Example 1 and 2, by formula (7) (circles), (15) (squares), (22) (triangles): a slowly (dashed) and rapidly (solid) varying Gaussian (top), a $C^{4}$ (dashed) and $C^{0}$ (solid) power distance function (bottom).
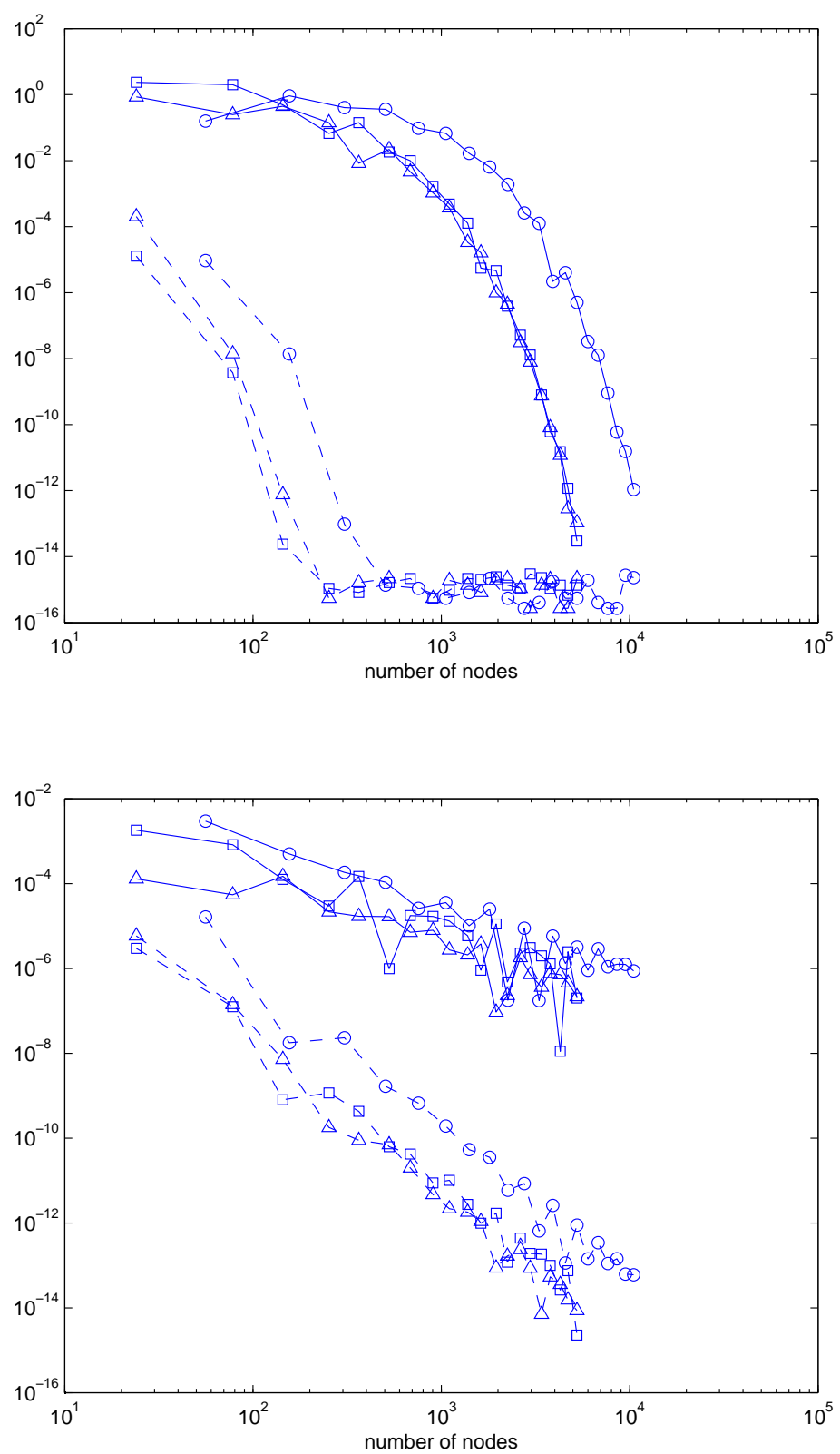
[5] G. Da Fies and M. Vianello, Trigonometric Gaussian quadrature on subintervals of the period, Electron. Trans. Numer. Anal. 39 (2012), $102-112$.

[6] G. Da Fies and M. Vianello, On the Lebesgue constant of subperiodic trigonometric interpolation, J. Approx. Theory 167 (2013), 59-64.

[7] G. Da Fies, A. Sommariva and M. Vianello, Matlab functions for subperiodic trigonometric quadrature and for product Gaussian quadrature on circular sections, online at:

http://www.math.unipd.it/ marcov/CAAsoft.html.

[8] W. Gautschi, Orthogonal Polynomials: Computation and Approximation, Oxford University Press, New York, 2004.

[9] W. Gautschi, Orthogonal polynomials (in Matlab), J. Comput. Appl. Math. 178 (2005), 215-234, software online at:

http://www.cs.purdue.edu/archives/2002/wxg/codes.

[10] W. Gautschi, Sub-range Jacobi polynomials, Numer. Algorithms 61 (2012), 649-657.

[11] S.G. Langton, The quadrature of lunes, from Hippocrates to Euler, Euler at 300, 5362, MAA Spectrum, Math. Assoc. America, Washington, DC, 2007.

[12] G. Meurant and A. Sommariva, Fast variants of the Golub and Welsch algorithm for symmetric weight functions, submitted (preprint online at: http://www.math.unipd.it/ marcov/CAApubl.html).

[13] W. Pleśniak, Multivariate polynomial inequalities via pluripotential theory and subanalytic geometry methods, Banach Center Publ. 72 (2006), 251-261.

[14] W. Pleśniak, Multivariate Jackson Inequality, J. Comput. Appl. Math. 233 (2009), 815-820.

[15] M.M. Postnikov, The problem of squarable lunes, American Mathematical Monthly 107 (2000), 645-651 (translated from the Russian by Abe Shenitzer).

[16] G. Santin, A. Sommariva and M. Vianello, An algebraic cubature formula on curvilinear polygons, Appl. Math. Comput. 217 (2011), 1000310015 .

[17] A. Sommariva and M. Vianello, Gauss-Green cubature and moment computation over arbitrary geometries, J. Comput. Appl. Math. 231 (2009), 886-896. 
[18] J. Stasica, The Whitney condition for subanalytic sets, Zeszyty Nauk. Uniw. Jagiellon. Prace Mat. 23 (1982), 211-221. 